In der Rubrik „Literatur kompakt" werden die wichtigsten Originalarbeiten aus der internationalen Fachliteratur referiert.

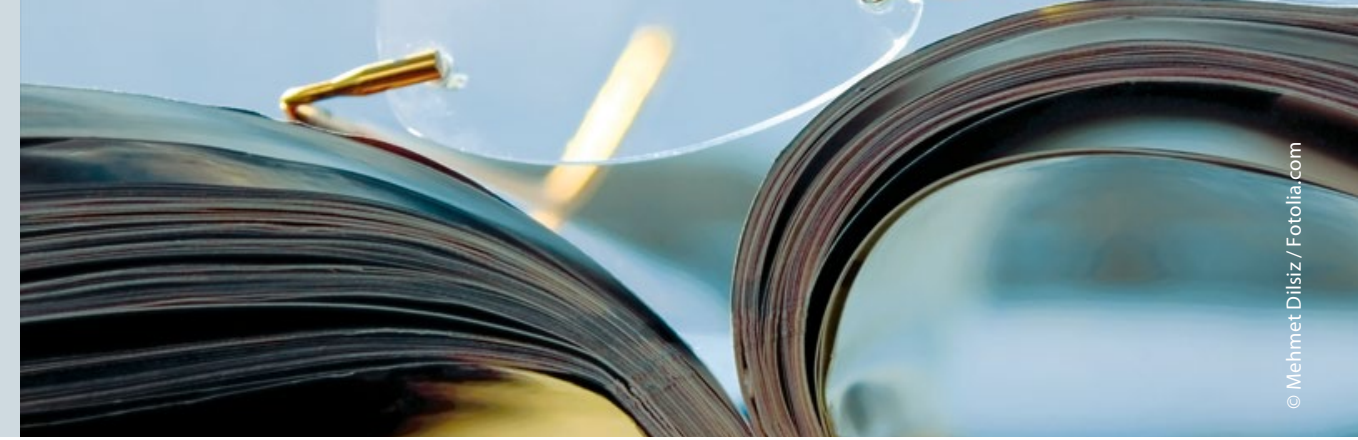

\section{MRT-Untersuchung: Geringes Risiko auch bei nicht MRT-fähigen Herzschrittmachern}

\begin{abstract}
Auch wenn ein Patient einen Schrittmacher oder Defibrillator ohne MRT-Zulassung trägt, können MRT-Untersuchungen außerhalb des Thorax durchgeführt werden, ohne den Patienten zu gefährden.
\end{abstract}

B ei Patienten mit implantierten Schrittmachern oder Defibrillatoren galten MRT-Untersuchungen lange Zeit als kontraindiziert. Dies war und ist Folge einer potenziellen Erwärmung von

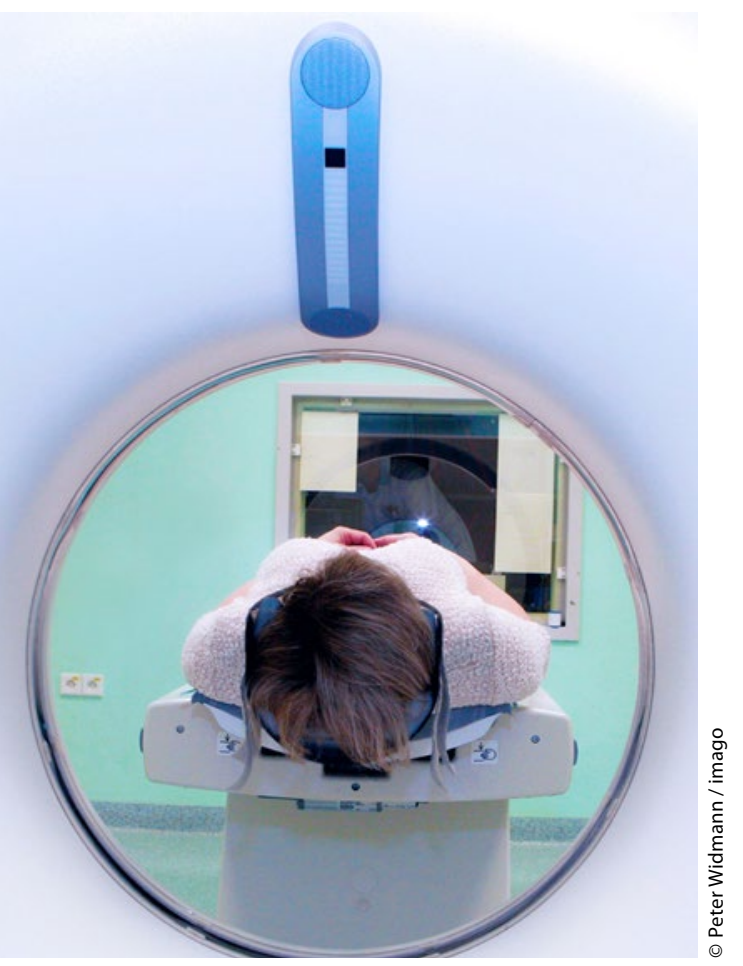

Eine MRT-Untersuchung kann bei wesentlich mehr Schrittmacherträgern erfolgen als bisher angenommen.
Sonden oder Veränderungen der Geräteprogrammierung unter dem Einfluss starker Magnetfelder. Prinzipiell waren bislang lediglich bei primär MRT-fähigen Geräten/Sonden mit entsprechender Zulassung MRT-Untersuchungen gestattet. Bisher war jedoch noch nicht klar, inwieweit bei entsprechenden Selektionierungsprozessen, MRT-Geräteeinstellungen und Geräteprogrammierungsalgorithmen MRT-Untersuchungen auch bei primär nicht MRT-fähigen Geräten möglich sind.

In den USA wurde daher in Kooperation mit der FDA ein großes prospektives Register zum besseren Verständnis der Risiken nicht MRT-fähiger Geräte bei 1,5-Tesla-MRT-Untersuchungen außerhalb des Thoraxraums initiiert. Im Rahmen des Registers wurde bei 1.000 Schrittmacher- und 500 DefibrillatorPatienten eine MRT-Untersuchung durchgeführt. Die Geräte wurden nach einem standardisierten Protokoll vor und nach der MRT-Untersuchung abgefragt und unter Umständen neu programmiert. Der primäre Endpunkt war definiert als Tod, Device- oder Sondenfehlfunktion, induzierte Arrhythmie, Verlust des „capture“ oder eine elektrische Reprogrammierung während der MRT-Untersuchung. Veränderungen in der Geräteprogrammierung zählten als sekundärer Endpunkt.
In der Gruppe aller Patienten gab es keine Todesfälle, keine Sondenfehler, keinen Verlust der Stimulierbarkeit und auch keine ventrikulären Arrhythmien. Ein ICD konnte nach der MRT-Untersuchung nicht ausgelesen werden, wobei dies schon vor der MRT-Untersuchung der Fall war.

Es wurden zudem sechs selbstlimitierende Episoden von Vorhofflimmern beobachtet, wie auch weitere sechs Fälle mit partiellem elektrischem Ausfall. Eine weitere MRT-Untersuchung führte nicht zu einer Erhöhung schwerwiegender Komplikationen.

Russo RJ et al. Assessing the Risks Associated with MRI in Patients with a Pacemaker or Defibrillator. N Engl J Med. 2017;376:755-764.

\section{Kommentar}

Die Daten des US-Registers zeigen, dass extrathorakale MRT-Untersuchungen auch bei Patienten mit nicht MRT-fähigen Schrittmachern und Defibrillatoren möglich sind, wenn entsprechende Protokolle zur Patientenauswahl sowie die Programmierung der Geräte vorgenommen werden. In enger Kooperation zwischen Radiologen und Kardiologen kann daher wesentlich mehr Schrittmacher- und Defibrillatorträgern eine MRT-Untersuchung angeboten werden als zuvor. Daten zu thorakalen MRTUntersuchungen liegen allerdings für diese Geräte-/Sondentypen noch nicht vor.

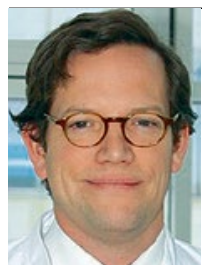

Prof. Dr. Peter W. Radke Klinik für Innere Medizin, Kardiologie, Schön Klinik Neustadt 